\title{
Spinal holocord epidural abscess evacuated with double thoracic interval laminectomy: a rare case report with literature review
}

\author{
Kaustubh Ahuja ${ }^{1} \cdot$ Lakshmana Das $^{1} \cdot$ Aakriti Jain ${ }^{1} \cdot$ Pradeep Kumar Meena $^{1} \cdot$ Shobha S. Arora ${ }^{1}$ Pankaj Kandwal ${ }^{1}$
}

Received: 17 May 2019 / Revised: 6 June 2019 / Accepted: 8 June 2019

(c) International Spinal Cord Society 2019

\begin{abstract}
Introduction Holocord spinal cord epidural abscess is an uncommon condition that may result in serious neurological complications. Prompt diagnosis and early treatment is of paramount importance for an optimum clinical outcome. This case report describes a novel technique of interval laminectomy at two sites in the thoracic spine and surgical decompression with the help of infant feeding tubes in a case of holocord spinal epidural abscess (HSEA).

Case presentation An 18-year-old male presented to the emergency department with high-grade fever and low back ache of 2 weeks duration and loss of bowel and bladder control for 4 days. Neurological examination revealed intact motor power and sensation in all four limbs at presentation; however, there was a rapid deterioration to complete quadriplegia within $24 \mathrm{~h}$. A diagnosis of holocord epidural abscess was made. Emergent decompression via interval thoracic laminectomy was done and appropriate antimicrobial therapy was instituted. At 10 months of follow-up, the individual showed complete neurological recovery. Discussion The technique used in this case is unique with respect to the level of laminectomy and the manoeuvre employed for pus evacuation. Complete neurological and functional recovery was achieved despite complete paralysis pre-operatively. The outcome indicates that there may be good prognosis for individuals with HSEA accompanied with neurological deficit and emergent surgical decompression.
\end{abstract}

\section{Introduction}

Spinal cord epidural abscess is an uncommon condition that may result in serious neurological complications. A true holocord spinal epidural abscess (HSEA) is even more infrequent and is defined as an abscess extending in the epidural region from the foramen magnum where the spinal and periosteal layers of the duramater fuse superiorly to the sacrococcygeal membrane inferiorly [1-3]. To the best of our knowledge, $<10$ cases of true HSEA have been reported. Even a small quantity of epidural collection can lead to rapid and profound neurological deterioration because the spinal canal is a closed compartment with limited epidural space. Prompt diagnosis and early treatment is of paramount importance for optimum clinical outcome and to prevent complications. Management for HSEA is controversial and generally includes

Kaustubh Ahuja

kaustubh1@live.in

1 Department of Orthopaedic Surgery, All India Institute of Medical Sciences, Rishikesh 249203 Uttarakhand, India a thorough surgical decompression with targeted antibiotic therapy. Widespread decompression and debridement can lead to complications like spinal instability, increased peri-operative blood loss, respiratory difficulties, prolonged recovery time and late-onset spinal deformities. This case report describes a novel technique of interval laminectomy at two sites in thoracic spine and surgical decompression with the help of infant feeding tubes in a case of HSEA.

\section{Case report}

An 18-year-old previously healthy male presented to the emergency department of AIIMS, Rishikesh, with high-grade fever and low back ache (visual analogue scale: 9/10) of 2 weeks duration and loss of bowel and bladder control for 4 days. There was no history of trauma or drug abuse. On clinical examination he was found to be febrile with severe tenderness and paraspinal muscle spasm throughout the dorsal and lumbar spine. Neurological exam revealed Medical Research Council (MRC) grade 5 power in all four limbs with intact sensation and absent reflexes (Fig. 1). Complete blood profile including viral markers and blood cultures were sent 


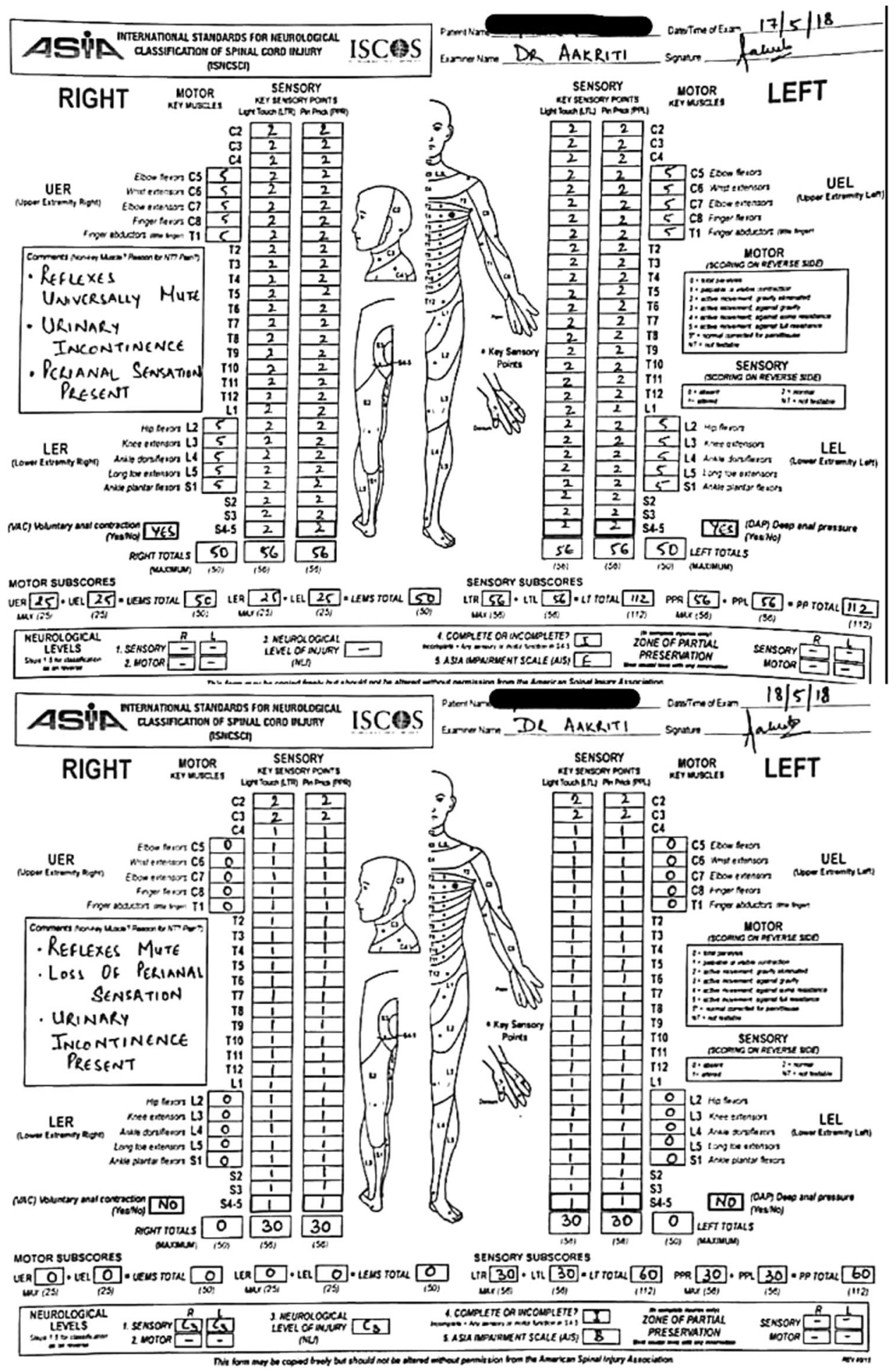

Fig. 1 Top: Neurological examination findings at the time of presentation. Bottom: Neurological presentation 24 h following admission showing complete quadriplegia

for testing. Whole spine radiographs and contrast-enhanced magnetic resonance (MR) imaging (MRI) was performed on emergency basis. Over the next $24 \mathrm{~h}$, his neurological status deteriorated rapidly with complete loss of muscle power throughout the upper and lower limbs and diminished sensation below the C3 dermatome. Neurological examination was recorded using international standards for the neurological classification of spinal cord injury (Fig. 1). Blood investigations revealed a total leucocyte count of $22,080 / \mathrm{mm}^{3}$ with neutrophilia, erythrocyte sedimentation rate (ESR) 
Fig. 2 Left: Pre-operative T2weighted sagittal (left) and axial (right) magnetic resonance (MR) images show epidural spinal abscess (white arrows) extending from $\mathrm{C} 1$ (top row) to S5 (bottom row) with a presacral abscess. Axial images show anteriorly pushed and compressed spinal cord. Right: One month post-operative T2weighted sagittal (left) and axial (right) MR images show complete resolution of epidural abscess with a healthy looking cord and rim of cerebrospinal fluid (white arrows) around the cord suggesting adequate decompression
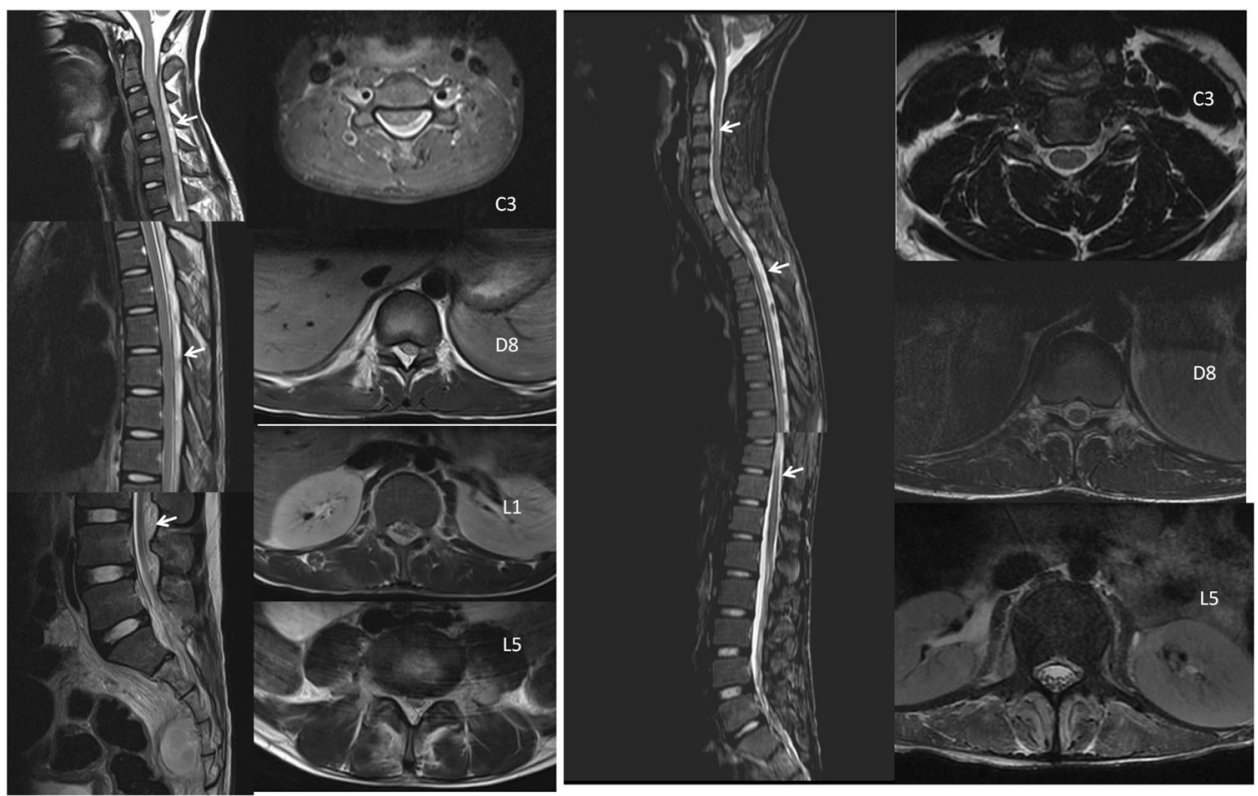

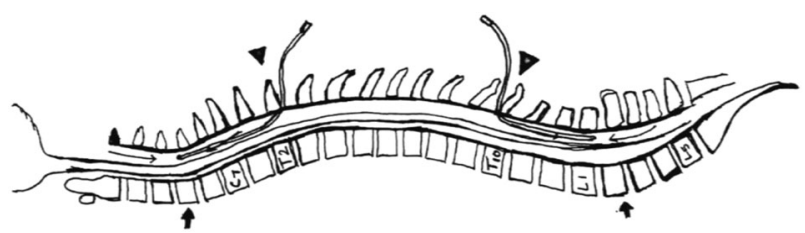

Fig. 3 Schematic diagram depicting the physiological 'troughs' of the human spine (thick arrows: apex of cervical and lumbar lordosis), thin arrows in the epidural space depicting the direction of gravitationassisted pooling of purulent material in the cervical and lumbar troughs in prone position, arrowheads depicting the planned laminectomy sites with feeding tubes inserted through these sites

$70 \mathrm{~mm} / \mathrm{h}$ and C-reactive protein (CRP) $44.8 \mathrm{mg} / \mathrm{L}$. The MRI scan showed a hyperintense epidural collection extending from C2 to sacral neural foramina with peripheral postcontrast enhancement causing anterior displacement of the cord (Fig. 2). A diagnosis of holocord epidural abscess was made and he was started on intravenous broad-spectrum antibiotics, including vancomycin, amikacin and metronidazole, along with supportive therapy, including analgesics and maintenance of hydration.

In view of his deteriorating neurologic status, he was planned for emergent decompression via laminectomy at $\mathrm{T} 2$ and T10 level. Intra-operatively, well-organized purulent material was found in the epidural space, which was loosely adherent to the underlying duramater. Infant feeding tubes of sequentially progressive sizes were used to evacuate pus and irrigate the canal. A controlled suction was applied on the feeding tube using a $20 \mathrm{~cm}^{3}$ syringe (Figs. 3, 4). Irrigation and evacuation procedures were continued alternatively till no purulent material was observed in the evacuated fluid. The two laminectomy sites were used to evacuate the epidural space along the entire length of spinal cord using feeding tubes. Following pus evacuation, the entire canal was irrigated using vancomycin solution. Culture from pus samples taken intraoperatively showed methicillin-resistant Staphylococcus aureus sensitive to linezolid and tetracycline. Intravenous linezolid was administered for 4 weeks following surgery. Adequate response to the antimicrobial therapy was confirmed by a decreasing trend of serial ESR, CRP and leucocyte count. Postoperatively physical rehabilitation and other supportive measures were initiated. On postoperative day 5, he showed improvement in muscle power in both upper limbs from MRC grade 0 to grade 3, which eventually improved to grade 4 over the next 2 weeks. Lower limb motor improvement was delayed and improved to grade 4 at the end of 4 weeks. Bowel and bladder incontinence persisted at the end of 4 weeks. Repeat MRI at the end of 4 weeks of antibiotic therapy showed no residual epidural collection and adequately decompressed spinal canal (Fig. 2). He was mobilized with the help of a walker and was discharged 4 weeks after surgery with a motor power of MRC grade 4 throughout all four limbs. Clean intermittent catheterization with special emphasis on perineal hygiene was taught at the time of discharge. Regular monthly follow-up visits were done with major emphasis on decreasing spasticity, bowel and bladder training and regaining full motor power. At the end of 10 months, the individual was re-evaluated and had normal motor and bowel and bladder function (Fig. 5). He was a community ambulator and was able to carry out all his activities of daily living independently. 


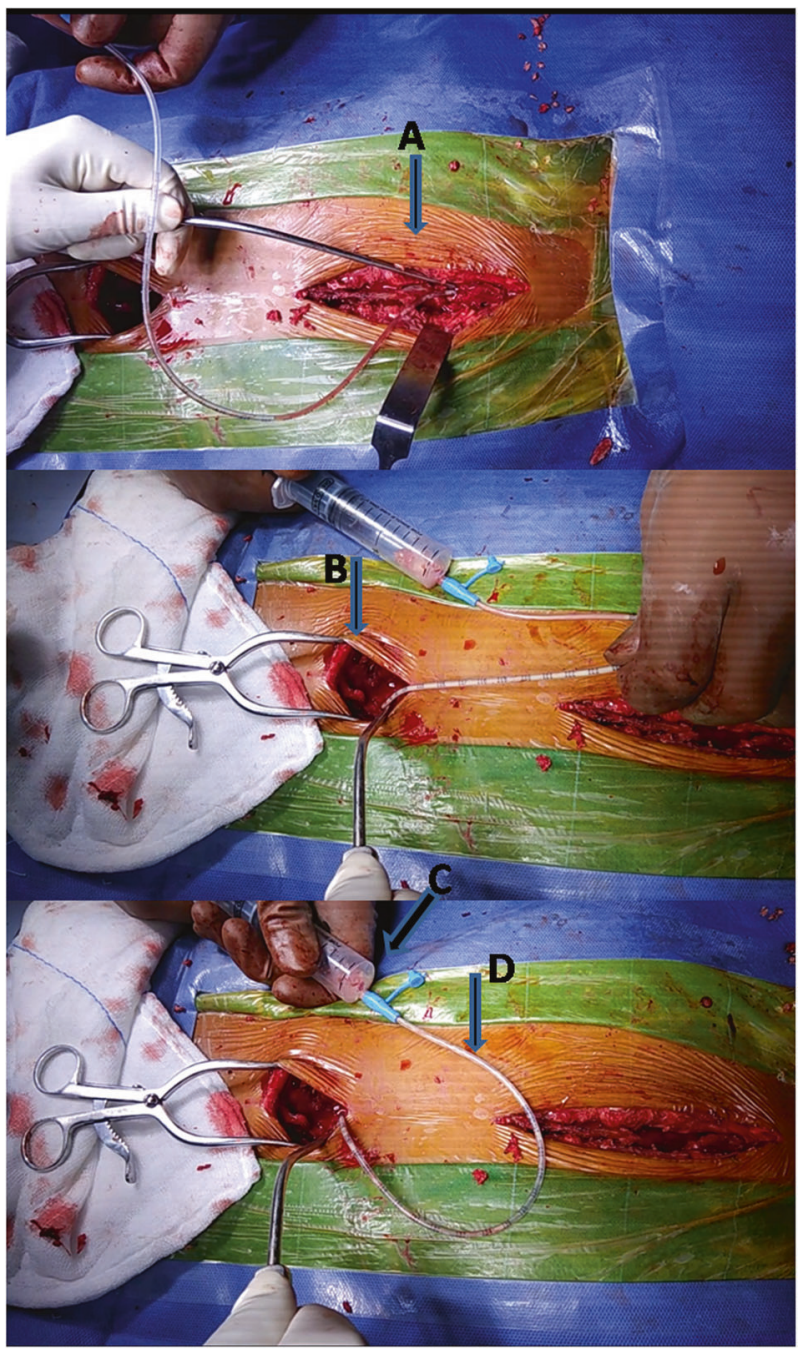

Fig. 4 Intraoperative images showing a proximal laminectomy site, b distal laminectomy site, c $20 \mathrm{ml}$ syringe applying controlled pressure over suction tube, and $\mathbf{d}$ white coloured pus being evacuated using the feeding tube

\section{Discusssion}

Spinal epidural abscess (SEA) is a rare but lethal condition. Its reported incidence ranges from 0.2 to $1.2 \%$ per 10,000 hospital admissions [4]. HSEA is an even rarer condition associated with high morbidity and mortality. Despite the advances in radiology, antibiotic therapy and surgical techniques, the mortality rate associated with HSEA is around $15 \%[5,6]$.

SEA can affect any age group with risk factors being diabetes, previous spinal surgery or intervention, intravenous drug abuse, malignancy and other immunocompromised states [1, 7]. Although a majority of individuals presenting with SEA have associated risk factors, $10-15 \%$ show no risk factors. SEA develops due to the seeding of microorganisms in the epidural space via hematogenous spread from other infective foci, for example, skin, soft tissue, lungs or direct inoculation, for example, spinal/epidural injection or spread from an adjacent focus, for example, psoas abscess and spondylodiscitis [8]. Hematogenous spread is the most common source accounting for over $50 \%$ of the cases [9]. In about $10 \%$ of cases, no source can be identified [9]. An important mode of spread of infection in the epidural region is through Batson's plexus that communicates with the venous drainage of pelvic organs. This valve-less plexus of venous channels may lead to seeding of microorganisms in the epidural region [10]. In our case, no risk factors or potential source of spread could be identified.

One of the causes for the high mortality associated with HSEA is the delay in diagnosis due to its variable presentation. HSEA most commonly presents with back pain, fever and neurological deficit. Heusner [11] has staged the clinical presentation of the disease in four stages based on progression of the disease. In the first stage the individual presents with back pain, fever and tenderness; the second stage is characterized by radicular pain, nuchal rigidity/neck stiffness and reflex changes; at the third stage, the individual presents with sensory-motor weakness and bowel and bladder dysfunction; the fourth stage presents with complete paralysis. The progression of the disease from stage one to four is rapid in these cases warranting the need for a high index of suspicion for early diagnosis and prompt management. Rapid progression of neurological deficit can be partly explained by the mechanism of deficit, which, apart from direct mechanical compression of the neural structures also includes neural insult via venous stasis and end-artery thrombosis leading to local circulatory disruption [12]. This case also demonstrated a rapid progression to stage four, that is, complete paralysis over a period of $24 \mathrm{~h}$ requiring emergent decompression.

Since the first successful decompressive laminectomy by Barth in 1901 for a patient with a thoracic epidural abscess, urgent surgical decompression with systemic antimicrobial therapy has been the gold standard treatment for management of SEA [13]. But with the advent of MRI, diagnosis of SEA in early stages, that is, before the onset of neurological deficit is now possible leading to an increased interest in conservative management of individuals with SEA without neurological deficit $[14,15]$. Numerous studies in the past two decades have seen an ongoing controversy over the optimal management of SEA [4, 16-19]. In a systematic review, Arko et al. [20] concluded that surgical intervention is indicated for individuals with paralysis of $<72 \mathrm{~h}$ duration, weakness, incontinence, instability and clinical deterioration, while medical management is appropriate for individuals with intact neurologic function, stable spine or paralysis more than $72 \mathrm{~h}$ duration. In this case, we planned urgent surgical decompression with systemic antibiotics due to the 
Fig. 5 Ten-month post-operative image of the Individual showing complete neurological recovery in bilateral upper and lower limbs

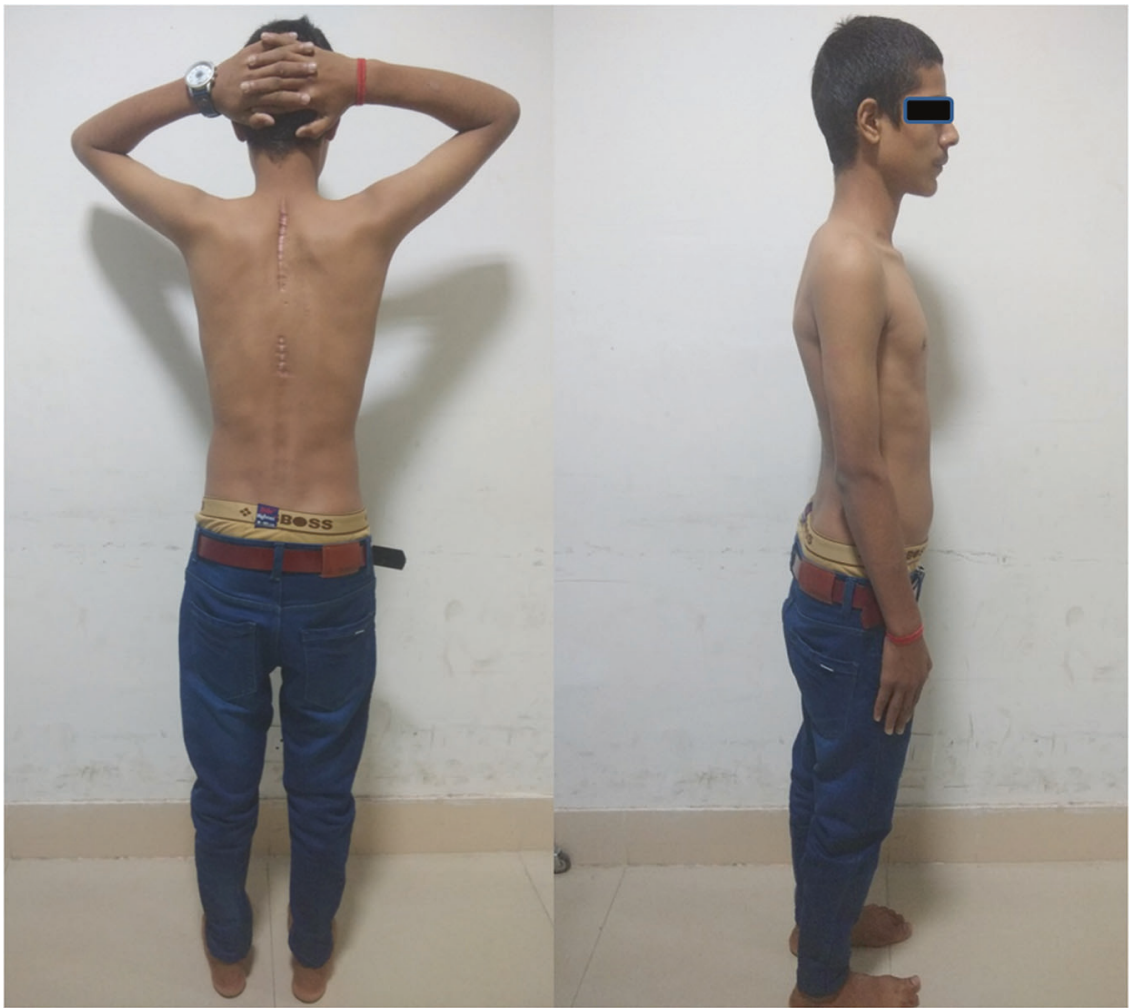

presence of bladder incontinence on presentation and worsening of neurologic function to complete paralysis within $24 \mathrm{~h}$ of presentation. Decompression of the entire spinal cord in a case of HSEA is ideally done by radical laminectomy of the entire spine with complete evacuation of the epidural pus and granulation tissue. However, this procedure puts the individual at risk of developing significant post-operative spinal instability and kyphosis without instrumentation [21]. Additionally, the morbidity associated with total spinal fusion with instrumentation cannot be underemphasized. A number of less extensive procedures have been described in an attempt to achieve adequate decompression while maintaining spinal stability. These include segmental or skip laminectomies with catheter irrigation [22-24], alternating side unilateral laminectomies with suction and irrigation [25] or focal laminectomy at the site of maximal cord compression [1]. Table 1 shows the described procedures and outcomes for true holocord epidural abscess over past 20 years. We planned to decompress the cord by interval laminectomies with pus evacuation using suction and irrigation with a flexible paediatric feeding tube. While deciding the levels of segmental laminectomies, the factors taken into consideration included thorough access to the entire spinal cord through the decided laminectomy levels, avoiding laminectomy at inherently unstable cervical and lumbar region, avoiding the apex of physiological kyphotic thoracic spine and avoiding disrupting the posterior tension band at the cervico-thoracic (C7-T1) and thoraco-lumbar (T12-L1) junction. We also considered the gravity assisted pooling of epidural pus in the 'troughs' of the human spine in prone position, that is, apex of physiological lordosis of cervical and lumbar spine (Fig. 3). We planned our segmental laminectomies as close to the troughs as possible. T2 and T10 were chosen as the laminectomy levels considering all the factors described above. Care was taken to preserve the facet joints so as to avoid any iatrogenic instability. For pus evacuation the earliest reports have described the usage of Fogarty catheter for mechanically draining the abscess [5]. We used semi-rigid infant feeding tubes with serially increasing sizes starting from 5-Fr to 8-Fr to facilitate blunt dissection through the organized purulent epidural material followed by irrigation and suction using warm saline, till suctioned material showed clear fluid. Unlike Fogarty catheter pus evacuation using feeding tube does not expose the cord to high pressure exerted by the inflated Fogarty tip [26]. Moreover, infant feeding tube is an inexpensive alternative to Fogarty catheter in a set up with limited resources.

The case presented here is peculiar because of a number of features. First, no risk factors could be identified. The individual was young without predisposing risk factors for HSEA. Second, the technique used in this case is unique with respect to the level of laminectomy and manoeuvre employed for pus evacuation. Complete neurological and functional recovery was achieved despite complete paralysis pre-operatively (stage 4). The outcome indicates there may be good prognosis for individuals with HSEA 


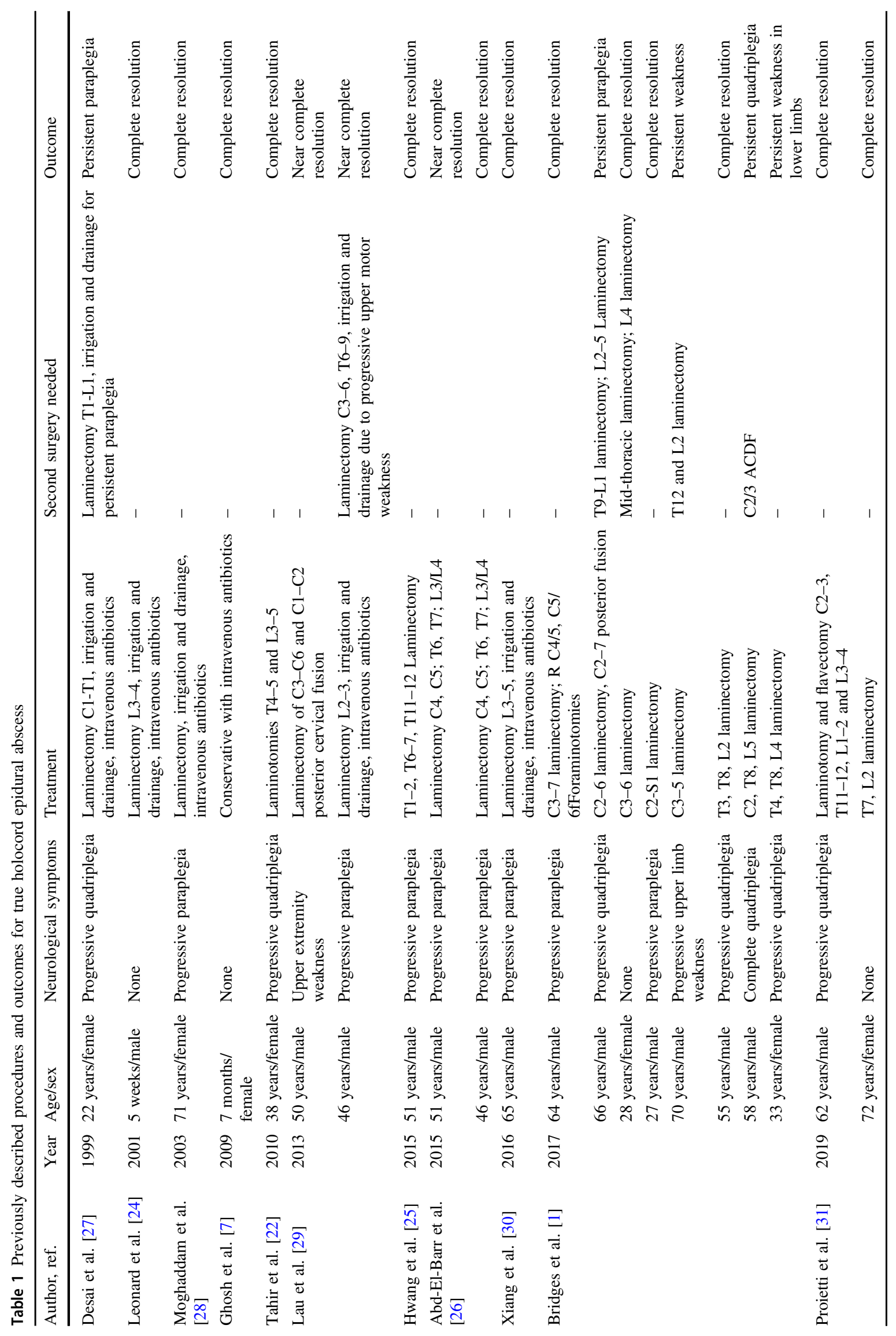


accompanied with neurological deficit and emergent surgical decompression.

Acknowledgements This work was Proofread by Ms. Rupin Mahiyaria.

\section{Compliance with ethical standards}

Conflict of interest The authors declare that they have no conflict of interest.

Consent for publication Written and informed consent was taken from the patient for using his picture for research purposes including publishing in research articles.

Publisher's note: Springer Nature remains neutral with regard to jurisdictional claims in published maps and institutional affiliations.

\section{References}

1. Bridges KJ, Than KD. Holospinal epidural abscesses-institutional experience. J Clin Neurosci. 2018;48:18-27.

2. Gala FB, Aswani Y. Imaging in spinal posterior epidural space lesions: a pictorial essay. Indian $J$ Radiol Imaging. 2016;26:299.

3. Richardson J, Groen GJ. Applied epidural anatomy. Contin Educ Anaesth Crit Care Pain. 2005;5:98-100.

4. Nussbaum ES, Rigamonti D, Standiford H, Numaguchi Y, Wolf AL, Robinson WL. Spinal epidural abscess: a report of 40 cases and review. Surg Neurol. 1992;38:225-31.

5. Schultz KD, Comey CH, Haid RW. Technical note. Pyogenic spinal epidural abscess: a minimally invasive technique for multisegmental decompression. J Spinal Disord. 2001;14:546-9.

6. Ansari A, Davies DWL, Lohn JWG, Culpan P, Etherington G. Extensive spinal epidural abscess associated with an unremarkable recovery. Anaesth Intens Care. 2004;32:825-9.

7. Sekhar Ghosh P, Loddenkemper T, Buitrago Blanco M, Marks M, Sabella C, Ghosh D. Holocord spinal epidural abscess. J Child Neurol. 2009;24:768-71.

8. Bond A, Manian FA. Spinal epidural abscess: a review with special emphasis on earlier diagnosis. BioMed Res Int. 2016;2016:1614328.

9. Darouiche RO. Spinal epidural abscess. N Engl J Med. 2006;355:2012-20.

10. Cheung WY, Luk KDK. Pyogenic spondylitis. Int Orthop. 2012;36:397-404.

11. Heusner AP. Nontuberculous spinal epidural infections. N Engl J Med. 1948;239:845-54.

12. Shah NH, Roos KL. Spinal epidural abscess and paralytic mechanisms. Curr Opin Neurol. 2013;26:314.

13. Reihsaus E, Waldbaur H, Seeling W. Spinal epidural abscess: a meta-analysis of 915 Individuals. Neurosurg Rev. 2000;23:175-204. discussion 205.

14. Savage JW, Moore TA, Arnold PM, Thakur N, Hsu WK, Patel $\mathrm{AA}$, et al. The reliability and validity of the thoracolumbar injury classification system in pediatric spine trauma. Spine (Phila $\mathrm{Pa}$ 1976). 2015;40:E1014-18.

15. Siddiq F, Chowfin A, Tight R, Sahmoun AE, Smego RA. Medical vs surgical management of spinal epidural abscess. Arch Intern Med. 2004;164:2409-12.

16. Rigamonti D, Liem L, Sampath P, Knoller N, Namaguchi Y, Schreibman DL, et al. Spinal epidural abscess: contemporary trends in etiology, evaluation, and management. Surg Neurol. 1999;52:189-96. discussion 197.

17. Adogwa O, Karikari IO, Carr KR, Krucoff M, Ajay D, Fatemi P, et al. Spontaneousspinal epidural abscess in Individuals 50 years of age and older: a 15-year institutional perspective and review of the literature: clinical article. J Neurosurg Spine. 2014;20:344-9.

18. Chen S-H, Chang W-N, Lu C-H, Chuang Y-C, Lui C-C, Chen S$\mathrm{F}$, et al. The clinical characteristics, therapeutic outcome, and prognostic factors of non-tuberculous bacterial spinal epidural abscess in adults: a hospital-based study. Acta Neurol Taiwan. 2011;20:107-13.

19. Connor DE, Chittiboina P, Caldito G, Nanda A. Comparison of operative and nonoperative management of spinal epidural abscess: a retrospective review of clinical and laboratory predictors of neurological outcome. J Neurosurg Spine. 2013;19:119-27.

20. Arko L, Quach E, Nguyen V, Chang D, Sukul V, Kim B-S. Medical and surgical management of spinal epidural abscess: a systematic review. Neurosurg Focus. 2014;37:E4.

21. Richmond BK, Schmidt JH. Seventeen level laminectomy for extensive spinal epidural abscess: case report and review. W V Med J.1994;90:468-71.

22. Tahir MZ, Hassan RU, Enam SA. Management of an extensive spinal epidural abscess from $\mathrm{C}-1$ to the sacrum: case report. J Neurosurg Spine. 2010;13:780-3.

23. Smith C, Kavar B. Extensive spinal epidural abscess as a complication of Crohn's disease. J Clin Neurosci. 2010;17:144-6.

24. Leonard J, Kaufman B. Treatment of a holocord epidural abscess. Case Illus J Neurosurg. 2001;94(1 Suppl):179.

25. Hwang R, Yung BH, Sedney C, Miele VJ. Treatment of holocord spinal epidural abscess via alternating side unilateral approach for bilateral laminectomy. W V Med J. 2015;111:1416-8.

26. Abd-El-Barr MM, Bi WL, Bahluyen B, Rodriguez ST, Groff MW, Chi JH. Extensive spinal epidural abscess treated with "apical laminectomies" and irrigation of the epidural space: report of 2 cases. J Neurosurg Spine. 2015;22:318-23.

27. Desai KI, Muzumdar DP, Goel A. Holocord intramedullary abscess: an unusual case with review of literature. Spinal Cord. 1999;37:866-70.

28. Moghaddam AM, Kilincoglu BF, Atalay B, Caner H, Altinörs N. Holocord epidural abscess: case report. Adv Ther. 2003;20:324-8.

29. Lau D, Maa J, Mummaneni PV, Chou D. Holospinal epidural abscess. J Clin Neurosci. 2014;21:517-20.

30. Xiang H, Ma X, Shen N, Yue B, Zhang G, Chen B. Holocord spinal epidural abscess: case report and literature review. Orthop Traumatol Surg Res (OTSR). 2016;102:821-5.

31. Proietti L, Ricciardi L, Noia G, Barone G, Valenzi E, Perna A, et al. Extensive spinal epidural abscesses resolved with minimally invasive surgery: two case reports and review of the recent literature. Acta Neurochir Suppl. 2019;125:345-53. 